INPLASY

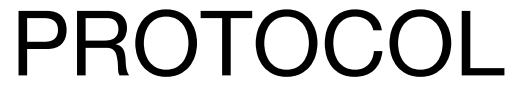

To cite: Guo et al. Salvia miltiorrhiza improves Type 2

Diabetes: A protocol for systematic review and metaanalysis. Inplasy protocol 2020110046. doi:

10.37766/inplasy2020.11.0046

Received: 11 November 2020

Published: 11 November 2020

Corresponding author:

Ying Guo

837404672@qq.com

Author Affiliation:

Chengdu University of

Traditional Chinese Medicine

Support: None.

Review Stage at time of this submission: Data analysis.

Conflicts of interest:

None.

\section{Salvia miltiorrhiza improves Type 2 Diabetes: A protocol for systematic review and meta-analysis}

Guo, Y1; Sun, J2; Zhang, R3; Yang, P4; Zhang, S5.

Review question / Objective: Are Salvia miltiorrhiza effective and safety in the treatment of Type 2 Diabetes?

Condition being studied: Diabetes refers to any group of metabolic diseases characterized by high blood sugar and generally thought to be caused by insufficient production of insulin, impaired response to insulin. Globally, patients with type 2 diabetes account for more than $85 \%$ of the total diabetic patients, and due to factors, such as obesity, aging, environment and lifestyle, the incidence of diabetes is rising. Salvia miltiorrhiza (SM) is a medicine used to treat diabetes in China. In recent years, it has been reported that SM has the effect of improving type 2 diabetes. However, there is no systematic review of its efficacy and safety yet. Therefore, we propose a systematic review to evaluate the efficacy and safety of SM for T2D.

INPLASY registration number: This protocol was registered with the International Platform of Registered Systematic Review and Meta-Analysis Protocols (INPLASY) on 11 November 2020 and was last updated on 11 November 2020 (registration number INPLASY2020110046).

\section{INTRODUCTION}

Review question / Objective: Are Salvia miltiorrhiza effective and safety in the treatment of Type 2 Diabetes?
Condition being studied: Diabetes refers to any group of metabolic diseases characterized by high blood sugar and generally thought to be caused by insufficient production of insulin, impaired response to insulin. Globally, patients with 
type 2 diabetes account for more than $85 \%$ of the total diabetic patients, and due to factors, such as obesity, aging, environment and lifestyle, the incidence of diabetes is rising. Salvia miltiorrhiza (SM) is a medicine used to treat diabetes in China. In recent years, it has been reported that SM has the effect of improving type 2 diabetes. However, there is no systematic review of its efficacy and safety yet. Therefore, we propose a systematic review to evaluate the efficacy and safety of SM for T2D.

\section{METHODS}

Participant or population: Patients diagnosed with Type 2 diabetes.

Intervention: Analyzed interventions included Salvia miltiorrhiza used as monotherapy, Chinese herbal compound prescription and related alternative therapies.

Comparator: Patients diagnosed with Type 2 Diabetes but who have not received it or who have only received nonpharmacological treatment.

Study designs to be included: Randomized controlled trial with no limitations on blinding or publication types will be included.

Eligibility criteria: Only randomized controlled trials of Salvia miltiorrhiza for the treatment of Alzheimer's disease will be includes. Collect only experiments recorded in Chinese and English. Non randomized trials, casereports, observational studies, and reviews will be excluded.

Information sources: We search the following databases from January 2010 to august 1, 2020: China National Knowledge Infrastructure, China Biological Medicine, Chinese Scientific Journals Data base, Wanfang database, PubMed, EMBASE. We search English and Chinese articles for review, and collect additional references from review references and original research articles.

Main outcome(s): The primary outcomes include 1. 2 hour plasma glucose 2. Fasting plasma glucose 3 . Hemoglobin 4 . Homeostasis model assessment of insulin resistance 5. Fasting plasma insulin and adverse events.

Quality assessment / Risk of bias analysis: Two reviews will assess the risk of publication bias for every included RCT with the Cochrane Risk of Bias Tool independently in terms of seven items, including random sequence generation, allocation concealment, blinding of participants and researchers, incomplete outcome data, selective reporting bias, and other bias. Each item will be graded as high, unclear, or low risk of bias. Inconsistency will be solved by consultation with a third reviewer.The Grading of Recommendations Assessment, Development and Evaluation (GRADE) guidelines will be utilized to grade the quality of evidence as very low, low, moderate, or high.

Strategy of data synthesis: In line with the Cochrane guideline, a fixed-effect model will be utilized to pool and analyze the outcome data if $12<50$, and a randomeffect model will be employed if $12 \geq 50$. Subgroup analysis or meta-regression will be performed to assess the potential sources and presentreasonable explanations for the heterogeneity.

Subgroup analysis: If eligible, a subgroup analysis will be performed to identify differences, including: country, gender, age, disease, degree, and complications.

Sensibility analysis: Sensitivity analysis will be applied to evaluate the stability of the pooled results of included RCTs according to the methodological quality, sample size and missing data.

Country(ies) involved: China. 
Keywords: Type 2 diabetes, Salvia miltiorrhiza, meta-analysis, protocol, randomized controlled trials, systematic review.

Contributions of each author:

Author 1 - Ying Guo.

Author 2 - Jianfeng Sun.

Author 3 - Renyan Zhang.

Author 4 - Peng Yang.

Author 5 - Sanyin Zhang. 